\title{
The Mu class glutathione transferase is abundant in striated muscle and is an isoform-specific regulator of ryanodine receptor calcium channels
}

\author{
Yasser Abdellatif, Dan Liu, Esther M. Gallant, Peter W. Gage, \\ Philip G. Board, Angela F. Dulhunty* \\ Division of Molecular Bioscience, John Curtin School of Medical Research, Australian National University, \\ P.O. Box 334, Canberra City, ACT 2601, Australia
}

Received 7 April 2006; received in revised form 14 July 2006; accepted 10 August 2006

Available online 4 October 2006

\begin{abstract}
Members of the glutathione transferase (GST) structural family are novel regulators of cardiac ryanodine receptor (RyR) calcium channels. We present the first detailed report of the effect of endogenous muscle GST on skeletal and cardiac RyRs. An Mu class glutathione transferase is specifically expressed in human muscle. An hGSTM2-2-like protein was isolated from rabbit skeletal muscle and sheep heart, at concentrations of $\sim 17-93 \mu \mathrm{M}$. When added to the cytoplasmic side of RyRs, hGSTM2-2 and GST isolated from skeletal or cardiac muscle, modified channel activity in an RyR isoform-specific manner. High activity skeletal RyR1 channels were inactivated at positive potentials or activated at negative potentials by hGSTM2-2 $(8-30 \mu \mathrm{M})$. Inactivation became faster as the positive voltage was increased. Channels recovered from inactivation when the voltage was reversed, but recovery times were significantly slowed in the presence of hGSTM2-2 and muscle GSTs. Low activity RyR1 channels were activated at both potentials. In contrast, hGSTM2-2 and GSTs isolated from muscle (1-30 $\mu \mathrm{M})$ in the cytoplasmic solution, caused a voltage-independent inhibition of cardiac RyR2 channels. The results suggest that the major GST isoform expressed in muscle regulates $\mathrm{Ca}^{2+}$ signalling in skeletal and cardiac muscle and conserves $\mathrm{Ca}^{2+}$ stores in the sarcoplasmic reticulum.
\end{abstract}

(C) 2006 Elsevier Ltd. All rights reserved.

Keywords: Muscle; Calcium signalling; Glutathione transferase; Ryanodine receptor

\section{Introduction}

The ryanodine receptor (RyR) $\mathrm{Ca}^{2+}$ release channel, located in the membrane of the internal sarcoplasmic reticu$\operatorname{lum}(\mathrm{SR}) \mathrm{Ca}^{2+}$ store, is central to $\mathrm{Ca}^{2+}$ signalling and contraction in skeletal and cardiac muscle. The RyR forms the hub of a giant macromolecular complex, which includes associated regulatory proteins such as calmodulin, FK506 binding proteins, Homer, sorcins and anchored kinases on the cytoplasmic side [1,2]. Within the SR, the luminal domains of the RyR bind to the membrane spanning proteins, triadin and junctin, which also associate with the $\mathrm{Ca}^{2+}$ binding protein calsequestrin [3]. This protein complex interacts with many ions and soluble proteins that are present in the SR lumen and cytoplasm of the muscle fibre. As a result of these inter-

\footnotetext{
* Corresponding author. Tel.: +6126125 4491; fax: +61 261254761

E-mail address: angela.dulhunty@anu.edu.au (A.F. Dulhunty).
}

actions, the ion channel can integrate information about the state of the cellular environment and accordingly adjust $\mathrm{Ca}^{2+}$ release from the sarcoplasmic reticulum, either to conserve the store or to boost contraction.

We have recently discovered that several members of the glutathione transferase (GST) structural family, which are present in the cytoplasm of skeletal and cardiac muscle, are novel modulators of RyR activity [4-6]. Although members of the GST family are normally considered to be detoxifying enzymes, new roles for previously well-described GSTs are being discovered, as well as new GSTs with novel catalytic activities and functions [7]. Some GSTs interact with protein kinases and may modulate signal transduction pathways or catalyse isomerization reactions between metabolic intermediates [8-10]. These roles are in addition to the recently discovered RyR calcium release channel regulation. The predominant GST expressed in human skeletal and cardiac muscle is hGSTM2-2, a member of the Mu class [11-13]. 
The ability of this class of GST to modulate RyR activity has not yet been tested and since hGSTM2-2 (previously termed GST4) is abundant and specifically expressed in striated muscle tissue [11] we have investigated its ability to interact with RyR channels isolated from skeletal muscle and heart. In addition we have investigated the distribution of hGSTM2-2 like enzymes in these tissues from rabbit and sheep, respectively.

The results show that a GSTM2-2 like enzyme is present in significant amounts $(\sim 17-93 \mu \mathrm{M})$ in the cytoplasm of rabbit skeletal muscle and sheep cardiac muscle. We show that human GSTM2-2 (hGSTM2-2) as well as GST isolated from both skeletal and cardiac muscle modulates RyR activity in a RyR-specific manner, causing activation of the skeletal RyR1 (with an activity-dependent inactivation at positive potentials) while, like other members of the GST structural family, it inhibits the cardiac RyR2 channel. The results provide strong evidence that the GST proteins present in the cytoplasm can significantly modulate $\mathrm{Ca}^{2+}$ signalling in skeletal and cardiac muscle.

\section{Methods}

\subsection{SR vesicle preparation}

Back and leg muscle was removed from New Zealand male rabbits and SR vesicles were prepared from sucrose gradient fractions using methods of [14] with minor changes [15]. SR vesicles were obtained from sheep heart using methods of [16] with minor modifications [17]. Vesicles were stored either in liquid $\mathrm{N}_{2}$ or at $-70^{\circ} \mathrm{C}$.

\subsection{GST activity in cytoplasm and membrane fractions of skeletal and cardiac muscle}

GST activity was measured in rabbit skeletal and sheep cardiac muscle. Cytosolic GST was estimated in the supernatant following homogenisation and centrifugation to remove tissue debris. GST activity was detected in skeletal muscle sucrose gradient fractions [14] and the P4 cardiac muscle pellet [16]. GST activity was measured using the substrate 1-chloro-2,4-dinitrobenzene (CDNB) [18]. In a buffer system containing $(\mathrm{mM}): 150 \mathrm{KCl}, 20 \mathrm{MOPS}$ and 20 imidazole $\mathrm{pH} 7.2$, to mimic physiological $\mathrm{pH}$ and salt content. The assays were performed in a Cary spectrophotometer (Varian, USA) at $25^{\circ} \mathrm{C}$ and a wavelength of $340 \mathrm{~nm}$. The reaction was followed for $5 \mathrm{~min}$. The calculated reaction rates were corrected for the non-specific enzyme activity using controls obtained with the buffer system alone.

\subsection{Isolation of muscle GST}

GSTs were purified by affinity chromatography using glutathione agarose beads. Cytosolic fractions were incubated with the beads overnight at $4{ }^{\circ} \mathrm{C}$. The beads were collected by centrifugation at $805 \times g$ for $5 \mathrm{~min}$, washed twice with a buffer containing $20 \mathrm{mM}$ Tris $\mathrm{pH}$ 7.3. Bound enzyme was eluted using a buffer containing $20 \mathrm{mM}$ Tris pH 9.6 and $10 \mathrm{mM}$ GSH. Free GSH was removed by dialysis against a buffer containing $(\mathrm{mM})$ : $150 \mathrm{KCl}, 20$ imidazole, $20 \mathrm{MOPS} \mathrm{pH} \mathrm{7.2,} \mathrm{overnight} \mathrm{at}$ $4{ }^{\circ} \mathrm{C}$.

\subsection{Electrophoresis and Western blots}

Electrophoresis of purified GSTs from muscle fibres and recombinant hGSTM2-2 was carried out on $12.5 \%$ SDSPAGE. Molecular weight standards and samples were diluted in a sample loading buffer containing (\%, w/v): 2.5 SDS, 10 glycerol, 5 mercaptoethanol, 0.02 bromophenol blue and $62.5 \mathrm{mM}$ Tris-HCl, $\mathrm{pH}$ 6.8. The electrophoresis buffer contained in mM: 25 Tris, 191 glycine and $0.1 \%$ (w/v) SDS. Gels were stained with Coomassie Blue. Western blots were performed as described by Towbin et al. [19]. Antisera against recombinant hGSTA1-1 (1:1000), hGSTA4-4 (1:1000), hGSTM2-2 (1:1000) and hGSTP1 (1:1000) were raised in rabbits in the laboratory of Prof. Philip Board (Australian National University, Canberra, Australia) and antiserum against rGSTM1-1 (1:1000) was provided by Prof. John Hayes (University of Dundee, Dundee, UK). The antibodies were diluted in PBS with $1 \%$ skim milk. After $1 \mathrm{~h}$ incubation with the primary antibody and washing, the membrane was incubated with peroxidase-conjugated goat-rabbit immunoglobulins antiserum (DAKO, 1:8000) for $1 \mathrm{~h}$ and the washed three times with PBS. Bound antibodies were visualized by using enhanced chemiluminescence (ECL, Amersham).

\section{5. hGSTM2-2 expression and purification}

Recombinant hGSTM2-2 was prepared according to [18] with some modifications. An E. coli (TG1) clone containing the hGSTM2-2 expression plasmid was grown to an $\mathrm{OD}_{555}$ of $\sim 0.6$ in $500 \mathrm{ml}$ of Luria broth (LB) culture media containing $0.1 \mathrm{mg} / \mathrm{ml}$ ampicillin (Sigma-Aldrich, USA). hGSTM2-2 expression was induced with $1 \mathrm{mM}$ IPTG (isopropyly- $\beta$-D-thiogalactopyrinoside). Bacteria were collected by centrifugation at $2000 \times g$ for $20 \mathrm{~min}$. The pellet was dispersed by mild sonication for $20 \mathrm{~s}$ in a buffer containing $20 \mathrm{mM}$ Tris $\mathrm{pH} 7.3$ and $0.1 \mathrm{mg} / \mathrm{ml}$ lysozyme (Branson Sonifer 250) and lysed at room temperature for $1 \mathrm{~h}$. The lysate was further sonicated and then centrifuged at $20,000 \times g$. The supernatant was collected and incubated with glutathione agarose beads overnight at $4{ }^{\circ} \mathrm{C}$. The beads where then centrifuged at $805 \times g$ for $5 \mathrm{~min}$, washed twice with $20 \mathrm{mM}$ Tris, pH 7.3. Bound GST was eluted with $20 \mathrm{mM}$ Tris $\mathrm{pH} 9.6$ and $10 \mathrm{mM}$ GSH. The eluted protein was assayed for activity using CDNB and purity was assessed by SDS $12.5 \%$ polyacrylamide gel electrophoresis. 


\subsection{Lipid bilayers and single channel recording and analysis}

Lipid bilayers were formed across a $150 \mathrm{~mm}$ aperture in a delrin cup, which separated cis (cytoplasmic) and trans (luminal) solutions. The lipid composition was phosphatidylethanolamine, phosphatidylserine and phosphatidylcholine $(5: 3: 2, \mathrm{v} / \mathrm{v})[15,17]$. SR vesicles $(50 \mu \mathrm{g})$ were added to the $c i s$ solution so that the cytoplasmic surface of the SR and RyRs faced that solution after incorporation. For SR vesicle incorporation, the standard solution compositions were: cis: $230 \mathrm{mM}$ CsMS, $20 \mathrm{mM} \mathrm{CsCl}, 5 \mathrm{mM} \mathrm{CaCl}_{2}$, and $10 \mathrm{mM}$ TES (pH 7.4); trans: $30 \mathrm{mM}$ CsMS, $20 \mathrm{mM}$ $\mathrm{CsCl}, 1 \mathrm{mM} \mathrm{CaCl}_{2}$, and $10 \mathrm{mM} \mathrm{TES} \mathrm{(pH} \mathrm{7.4).} \mathrm{For} \mathrm{recording,}$ the cis chamber was perfused with a standard cis solution containing either $100 \mu \mathrm{M} \mathrm{Ca}^{2+}$ or $100 \mathrm{nM} \mathrm{Ca}^{2+}$ (buffered with $2 \mathrm{mM}$ BAPTA). $\mathrm{Cs}^{+}$in the trans chamber, was raised to $250 \mathrm{mM}$ by the addition of $200 \mathrm{mM}$ CsMS. Single channel parameters were obtained using the program Channel 2 (developed by P.W. Gage and M. Smith, John Curtin School of Medical Research, Canberra, Australia). Channel activity was assessed from $30 \mathrm{~s}$ records and was expressed as relative open probability $\left(P_{\mathrm{o}}\right) . P_{\mathrm{o}}$ was measured in two ways, either by using the threshold discriminator, which is set above the baseline noise at $\sim 20 \%$ of maximal current $(I)$, or from fractional mean current $\left(I_{\mathrm{F}}^{\prime}\right)$, which is the average of all data points obtained during a recording period, divided by the maximum single channel current. $I_{\mathrm{F}}^{\prime}$ is approximately equal to the $P_{\mathrm{o}}$ measured by threshold discrimination when most of channel openings are to the maximum conductance. The cis chamber was held at virtual ground and the potential in the trans chamber controlled. All electrical potentials are expressed using standard physiological convention (i.e. cytoplasm relative to lumen). Single channel recordings were routinely obtained using bilayer potential differences of +40 or $-40 \mathrm{mV}$. When appropriate, the potential was varied between $0 \mathrm{mV}$ and +60 or $-60 \mathrm{mV}$. Measurements were carried out at $23 \pm 2{ }^{\circ} \mathrm{C}$.

In order to quantify inactivation, the time at which the current during the burst of activity immediately after the voltage pulse decreased by at least $50 \%$ of its original value, and remained below $50 \%$ for at least $1 \mathrm{~s}$, was measured and defined as the "inactivation time". Slow changes in baseline current, including the tail of the capacitive current were corrected using the program Baseline (written by DR Laver).

\subsection{Statistics}

Average data are presented as mean \pm S.E.M. The significance of differences between control and test values were tested using an analysis of variance (ANOVA, single factor or two-factor without replication. Two-factor was used unless otherwise stated). A Student's $t$-test for paired data or a sign test [20] was used as appropriate. A $P$-value of $<0.05$ was considered to be significant.

\section{Results}

\subsection{Mu class GST found in skeletal and cardiac muscle}

GST activity was assessed in sheep heart and rabbit skeletal muscle preparations. The species were chosen because RyR1 was isolated from rabbit back and leg muscle and RyR2 from sheep heart. Both the cytosolic and membrane fractions obtained from both skeletal and cardiac muscle showed significant activity with the GST substrate CDNB (see Section 2), indicating that GSTs were present in both fractions (Table 1 ). The activity in the cytoplasm was $\sim 10$ times greater than the activity in the membrane fractions and the activity in skeletal cytoplasm was significantly greater than that in cardiac cytoplasm.

GSTs were purified from the cytosolic fractions using affinity chromatography (see Section 2) with immobilised GSH. GSTs in skeletal and cardiac muscle are likely to be a mixture of different members of the $\mathrm{Mu}$ and Pi classes of GSTs as in human muscle [21]. Recombinant hGSTM2-2 and purified muscle GSTs were run on SDS-PAGE (Fig. 1A). The recombinant protein showed a main monomer band at $28.8 \mathrm{kDa}$ and a minor band at $57.9 \mathrm{kDa}$, that is probably crosslinked dimer. Purified GST preparations from sheep heart cytosol showed discrete bands at 28.8 and $25.5 \mathrm{kDa}$, while preparations from rabbit skeletal muscle formed one major broad band at $25.5 \mathrm{kDa}$ with minor components at 30 and $25 \mathrm{kDa}$. Dimers were not detected in the purified muscle GSTs. Western blots, using polyclonal antibodies against human (h) hGSTA1-1, hGSTA4-4, rat (r) rGSTM11, hGSTM2-2, and hGSTP1-1, showed that the glutathione affinity purified fraction from sheep cardiac muscle contained GSTs that cross reacted with anti-rGSTM1-1, anti-hGSTM22 and anti hGSTP1-1 antibodies, whereas the rabbit skeletal muscle GST preparation only cross reacted with antirGSTM1-1, anti-hGSTM2-2 but not with antibodies against GSTA1-1, GSTA4-4 and GSTP1-1 (Fig. 1B). Anti-rGSTM1-

Table 1

GST activity in the cytosol and membrane fractions isolated from rabbit skeletal and sheep cardiac muscle

\begin{tabular}{lc}
\hline & Activity $(\mathrm{nmol} / \mathrm{mg} / \mathrm{min})$ \\
\hline Skeletal muscle fraction & \\
Cytosol & $399.0 \pm 27$ \\
B1 & $32.2 \pm 7$ \\
B2 & $50.0 \pm 2$ \\
B3 & $42.3 \pm 2$ \\
B4 & $16.9 \pm 1$ \\
Cardiac muscle fraction & \\
Cytosol & $347.0 \pm 2$ \\
P4 & $16.9 \pm 1$ \\
\hline
\end{tabular}

GST activity was measured using the substrate CDNB (see Section 2). Sucrose density band B1 is enriched in T-tubule membrane, B2 in surface membrane, B3 in longitudinal SR and B4 in terminal cisternae membrane. Activity is significantly lower in B4. Cardiac muscle P4 contains elements of all membrane systems. Enzyme activity is given as mean \pm S.D. from five experiments. 


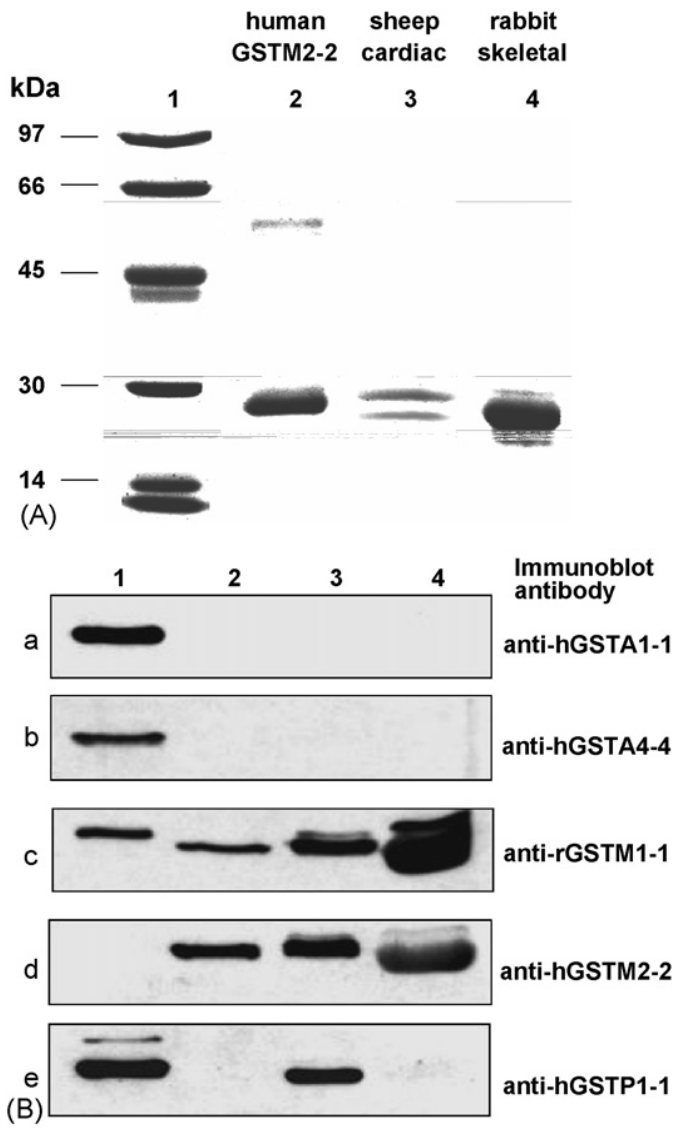

Fig. 1. GSTs, including an hGSTM2-2-like protein, are present in the cytosol of rabbit skeletal and sheep cardiac muscle fibres. (A) Coomassie Blue stained SDS-PAGE gel: lane 1, molecular size markers; lane 2, recombinant hGSTM2-2; lane 3, glutathione affinity purified GST fraction from homogenised sheep cardiac muscle fibres; lane 4 , glutathione affinity purified GST fraction from homogenised rabbit skeletal muscle fibres. (B) Western blots using different antibodies to identify GSTs of different classes present in the glutathione affinity purified fraction obtained from rabbit skeletal and sheep cardiac muscle fibres. Lane 1, recombinant human GSTA1-1, A4-4, M1-1 and P1-1 proteins as positive controls in panels a, b, c, and e, respectively, and blank as a negative control in panel $\mathrm{d}$; lane 2 , recombinant hGSTM2-2; lane 3, GST proteins affinity purified from sheep cardiac muscle cytosol; lane 4, GST proteins affinity purified from rabbit skeletal muscle cytosol.

1 antibody cross-reacted with recombinant hGSTM2 protein (Fig. 1B, panel c, lane 2). There are two proteins that cross-reacted with anti-rGSTM1-1 antibody in the GST fractions extracted from cardiac (correspondent with $29.2 \mathrm{kDa}$ $28.8 \mathrm{kDa}$ bands) or skeletal muscles (corresponding to $30 \mathrm{kDa}$ and $25.5 \mathrm{kDa}$ bands) (Fig. 1B, panel c, lanes 3 and 4). The top bands in each case reacted strongly only with anti-rGSTM1-1 antibody, indicating that they are likely to be a GSTM1-1-like proteins. The lower more abundant bands cross-reacted with both anti-rGSTM1-1 and anti-hGSTM2-2 antibody, suggesting that they are GSTM2-2-like proteins (Fig. 1B, panels c and d, lanes 3 and 4). The MW of the skeletal muscle GSTM22-like protein was slightly less than that of the recombinant hGSTM2-2 and the cardiac GSTM2-2-like protein, probably due to skeletal muscle-specific post-translational modifica- tion or species variation. The GSTP1-1 antibody cross reacted with $25.5 \mathrm{kDa}$ protein from cardiac muscle GST preparation, indicating that it is likely to be a Pi class GST. These results indicate that there is little if any Alpha class GST in these muscle preparations and that there is a small amount of $\mathrm{Pi}$ class GST in the affinity purified fraction from sheep heart. The intensity of staining and thickness of the bands in Fig. 1B suggests that the predominant GST in both muscle preparations is closely related to human GSTM2-2. This is consistent with the situation in humans.

The total amount of GST purified was $\sim 20 \mathrm{mg}$ from skeletal preparations or 3-4 mg from cardiac preparations. To calculate the cytoplasmic concentration we assume that the $150 \mathrm{~g}$ of muscle used has a volume $\sim 150 \mathrm{ml}$ [22]. Since $\sim 15 \%$ of this volume is occupied by mitochondria, nucleus and sarcoplasmic reticulum and $80 \%$ occupied by myofilaments [23] the remaining accessible cytoplasmic volume is $\sim 7.5 \mathrm{ml}$. Thus, the concentration of skeletal GST is close to $2.7 \mathrm{mg} / \mathrm{ml}$ and cardiac GST is $0.5 \mathrm{mg} / \mathrm{ml}$. Since the molecular weight of a GST subunit is approximately 28,800 , the total GST concentration in skeletal cytoplasm is $\sim 93 \mu \mathrm{M}$ and in cardiac cytoplasm is $\sim 17 \mu \mathrm{M}$. If GSTM2-2 exists as a dimer in the cytoplasm, then the dimer concentrations would be 46.5 and $8.5 \mu \mathrm{M}$, respectively.

\subsection{Effects of hGSTM2-2 on skeletal RyRI channels}

Initial experiments were performed with channels that were exposed to a cytoplasmic ( $c i s) \mathrm{Ca}^{2+}$ concentration of $100 \mu \mathrm{M}$. As previously described [24], these RyR1 channels fell into two sub populations, one with high activity (open probability $>0.05$ ) and one with lower activity (open probability <0.05). The effect of hGSTM2-2 depended on the initial activity of the channels. It reversibly activated the low activity channel shown in Fig. 2A and B at positive and negative potentials when added at $27 \mu \mathrm{M}$. The average data from five experiments showed significant activation at $+40 \mathrm{mV}$ and a trend towards activation at $-40 \mathrm{mV}$ that was not statistically significant. The initial $P_{\mathrm{o}}$ of these channels was $0.026 \pm 0.10$. Lower concentrations of hGSTM2-2 produced either no effect or a weak activation. Inhibition, typical of that seen with lower concentrations of the protein on RyR2 channels (see below) was not observed. In contrast to the low activity channels, hGSTM2-2 $(4-30 \mu \mathrm{M})$ added to the cis side of channels with high activity had a voltage-dependent action (Fig. 3). The mean current during $30 \mathrm{~s}$ recordings at $+40 \mathrm{mV}$ was reduced after adding hGSTM2-2 $(n=9)$. In contrast to $+40 \mathrm{mV}$, activity at $-40 \mathrm{mV}$ increased with hGSTM2-2 $(n=9)$. The effects at either potential were reversed when the potential was changed to the opposite polarity. Channel activity was restored to control levels when the GST was perfused out of the cis chamber $(n=5)$. The changes in activity were seen with $4 \mu \mathrm{M}$ hGSTM2-2 and maximum effects were seen with $8 \mu \mathrm{M}$ of protein. In five control experiments, $113 \mu \mathrm{l}$ of the vehicle for hGSTM2-2 (the volume of buffer normally added with $16 \mu \mathrm{M}$ GST) added alone did not alter activity. 

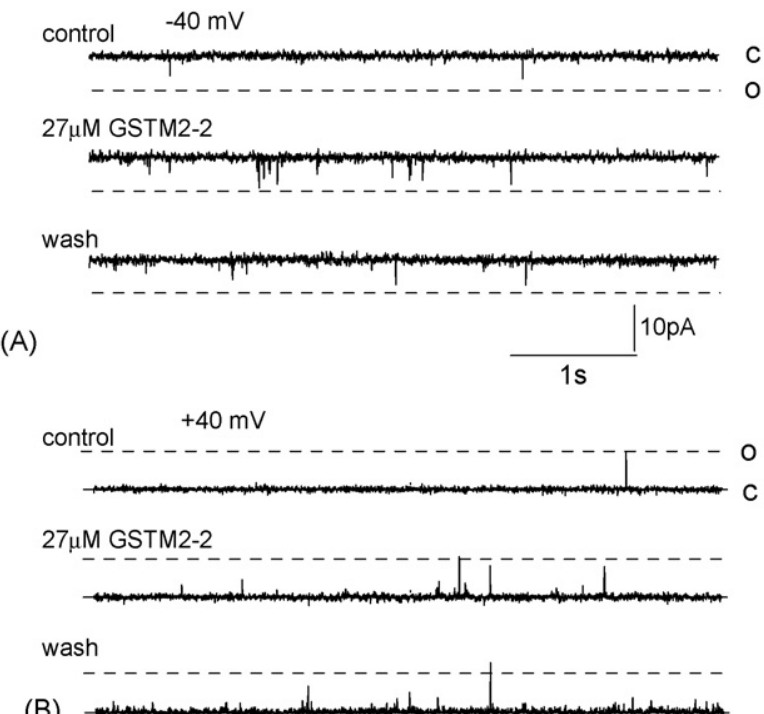

(B)
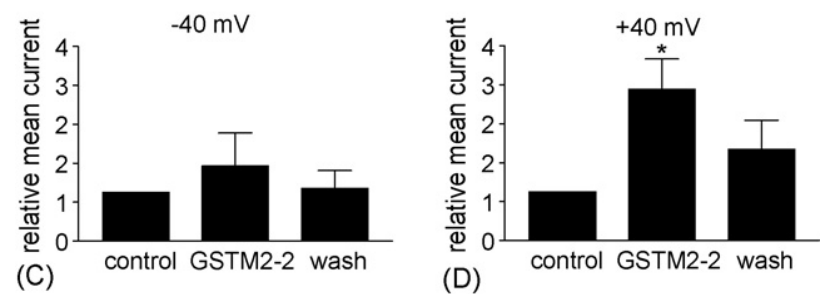

Fig. 2. Low activity RyR1 channels in lipid bilayers are activated at positive and negative potentials when $27 \mu \mathrm{M}$ hGSTM2-2 is added to the cytoplasmic solution. (A and B) Records from a bilayer containing one active RyR1 channel under control conditions (upper panel), after addition of $27 \mu \mathrm{M}$ hGSTM2-2 (middle panel) and after perfusion of the cis chamber (lower panel). Channel opening at $-40 \mathrm{mV}$ was downward (A), or upward at $+40 \mathrm{mV}$ (B) from the closed level (c, solid line) to the maximum open level (o, broken line). (C and D) Average relative open probability measured from $30 \mathrm{~s}$ records from low activity channels in five experiments at -40 and $+40 \mathrm{mV}$. The asterisk indicates a significant difference from control.

Channel activity returned to control levels when the GST was removed from the cis chamber by perfusion with 10 volumes of $c$ is solution lacking hGSTM2-2. As with the low activity channels, no evidence of the RyR2-like voltage-independent inhibition was seen in RyR1 channels exposed to hGSTM2-2 at any concentration.

The activity-dependent effects on RyR1 were also seen when hGSTM2-2 was added to channels that were relatively inactive in the presence of a sub-activating (resting) cytoplasmic $\mathrm{Ca}^{2+}$ concentration of $100 \mathrm{nM}$ (Fig. $4 \mathrm{~A}, n=6$ ). The channels with an initial open probability of $<0.001$, were not significantly activated by the hGSTM2-2. If the cis $\mathrm{Ca}^{2+}$ concentration was then increased to $100 \mu \mathrm{M}$, RyR activity increased to levels seen in high activity channels in the presence of hGSTM2-2 with $100 \mu \mathrm{M} \mathrm{Ca}^{2+}$ (compare Fig. 4B with Fig. 3 above). Therefore, the channels were less than normally activated by $\mathrm{Ca}^{2+}$ at $+40 \mathrm{mV}$ or more than normally activated at $-40 \mathrm{mV}$ (Fig. 4C). hGSTM2-2 was also effective in modifying RyR 1 channels that were activated by $2 \mathrm{mM}$ AMP-PNP (with $100 \mathrm{nM}$ cis $\mathrm{Ca}^{2+}$, open probability >0.01) in a voltage-dependent manner (Fig. 4C, $n=6$ ). If hGSTM22 was added to channels before AMP-PNP, the response to AMP-PNP was depressed at $+40 \mathrm{mV}$ or enhanced at $-40 \mathrm{mV}$ (Fig. 4D, $n=6$ ). Thus, channel activity with hGSTM2-2 plus AMP-PNP was independent of the order in which the compounds were added. Finally, when $2 \mathrm{mM} \mathrm{MgCl}_{2}$ was added in the presence of hGSTM2-2 plus AMP-PNP, channel activity fell to levels recorded with $100 \mathrm{nM}$ cis $\mathrm{Ca}^{2+}$ alone (Fig. 4E, $n=6$ ). In conclusion, the effects of hGSTM2-2 were apparent only in channels that were activated by $\mathrm{Ca}^{2+}$ or adenine nucleotides and the modulation was independent of the mechanism of activation.

\subsection{Voltage- and time-dependent effects of hGSTM2-2 on RyRl on high activity channels}

When high activity channels exposed to $100 \mu \mathrm{M}$ cis $\mathrm{Ca}^{2+}$ were modified by hGSTM2-2, their activity varied in a timedependent manner after a voltage pulse. This can be seen by comparing activity during the first second after the voltage step with activity during the entire $30 \mathrm{~s}$ recording period at each potential. At positive potentials, activity during the first second after the voltage step was similar to or greater than control in the presence of hGSTM2-2 (Fig. 5A), but then decreased rapidly (Fig. 5B). In contrast at negative potentials, activity was initially similar to or less than control during the first second (Fig. 5C), but then increased to a level that was maintained during the subsequent $30 \mathrm{~s}$ (Fig. 5D). Mean current was measured between $\sim 150 \mathrm{~ms}$ and $1 \mathrm{~s}$ after a voltage step (avoiding the transient capacitive current) and then during the subsequent $30 \mathrm{~s}$. The "inactivation time" (i.e. the time for current to decay to $50 \%$ of its initial value and to remain at that level for $>1 \mathrm{~s}$ ) was measured as an indicator of the rate of decay of the current at positive potentials. Inactivation times are indicated by the solid lines at the start of the records shown in Fig. 5B. On average, activity during the first second after the voltage step was no different from that in the absence of hGSTM2-2 at negative potentials, but tended to be greater than control at positive potentials, particularly +30 and $+40 \mathrm{mV}$ (Fig. 6A). This was in contrast to the average mean current over the $30 \mathrm{~s}$ recording period (Fig. 6B), which was greater than control between -20 and $-60 \mathrm{mV}$ and less than control between +20 and $+60 \mathrm{mV}$ (Fig. 6B).

The difference between activity at the beginning and end of the $30 \mathrm{~s}$ recording period after voltage steps to positive and negative potentials is illustrated again in Fig. 6C. The decay in activity with time after the voltage pulse to positive potentials is typical of voltage-dependent inactivation commonly seen in RyRs [17,25-29]. In the absence of hGSTM2-2, this inactivation at $+40 \mathrm{mV}$ was observed in $89 \%$ of records from channels activated by either $100 \mu \mathrm{M} \mathrm{Ca}^{2+}$ or by $2 \mathrm{mM}$ AMPPNP. The average inactivation time was $7.2 \pm 0.6 \mathrm{~s}(n=8)$. In contrast, after adding hGSTM2-2, inactivation at $+40 \mathrm{mV}$ was seen in $100 \%$ of records and the average inactivation time was reduced to $2.0 \pm 0.4 \mathrm{~s}(n=5)$ (Fig. 7). Inactivation was faster at $+50 \mathrm{mV}$ than $+40 \mathrm{mV}$ under control conditions $(2.9 \pm 0.7 \mathrm{~s}$, 

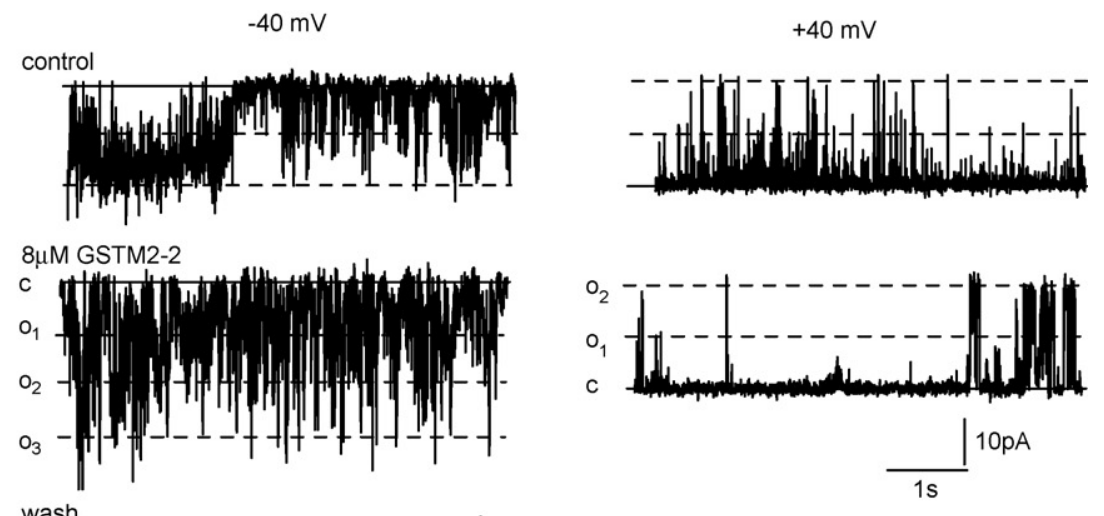

(A)
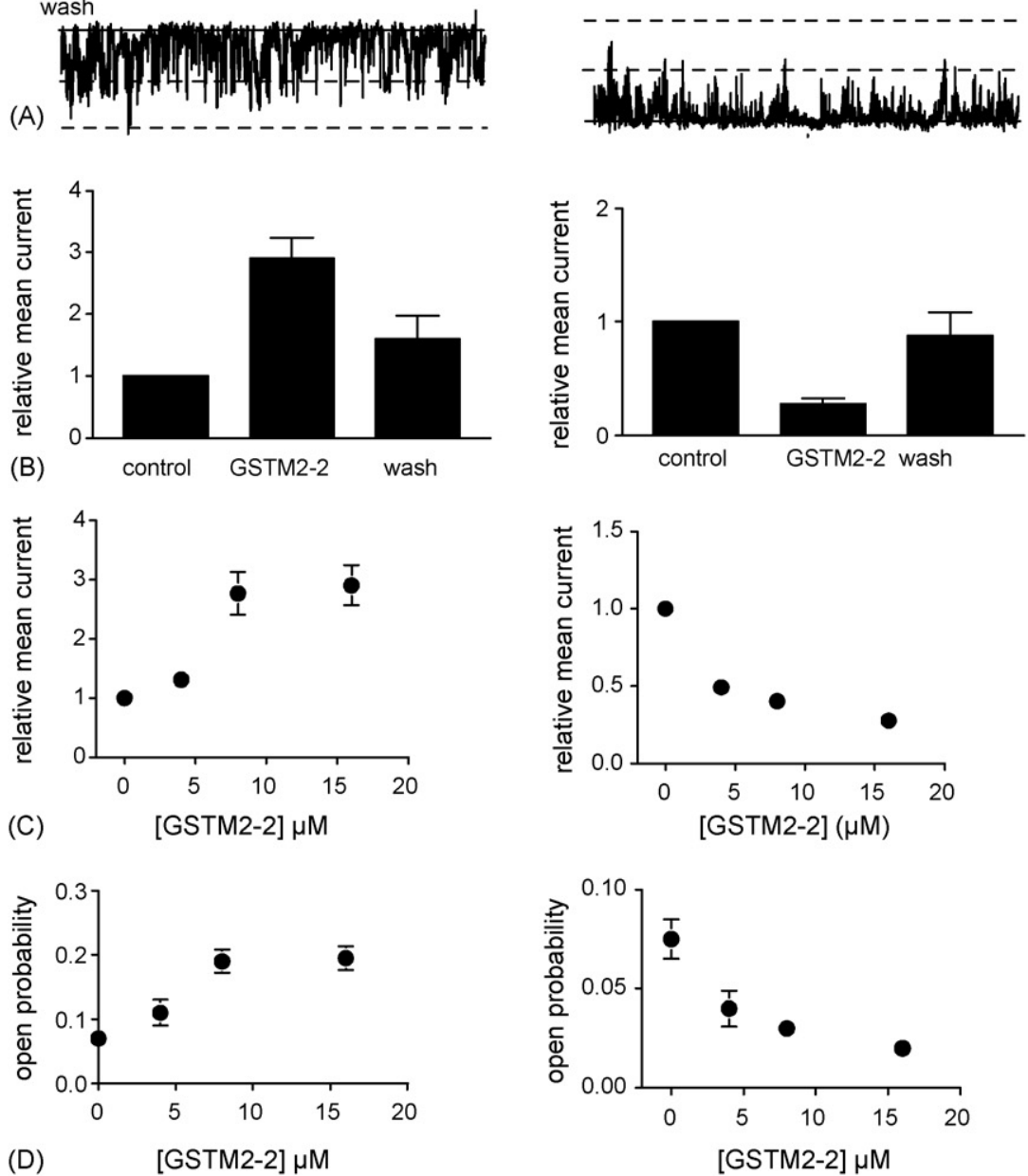

Fig. 3. hGSTM2-2 added to cytoplasmic (cis) solution causes an overall increase in the activity of high activity RyR1 channels at negative potentials or depresses activity at positive potentials, when channel activity is measured over the $30 \mathrm{~s}$ period after the voltage pulse. (A) Records from a bilayer containing three active RyR1 channels at $-40 \mathrm{mV}$ (left panel) or $+40 \mathrm{mV}$ (right panel), under control conditions (upper trace), with $8 \mu \mathrm{M} \mathrm{hGSTM} 2-2$ (middle trace) and after washout of the cis solution (lower trace). Channel opening is downward at $-40 \mathrm{mV}$, or upward at $+40 \mathrm{mV}$, from the closed level (c) to levels $\mathrm{o}_{1}, \mathrm{o}_{2}$ or $\mathrm{o}_{3}$ when one, two or three channels were open at one time. (B) Average relative mean current at $-40 \mathrm{mV}$ (left) or $+40 \mathrm{mV}$ (right) under control conditions, with 8-15 $\mu$ M hGSTM2-2 ( $n=9$ experiments) and after washout $(n=5)$. (C and D) Average relative mean current (C) or open probability (D) at $-40 \mathrm{mV}$ (left) or $+40 \mathrm{mV}$ (right) as a function of hGSTM2-2 concentration (each point is the average of at least five observations).

$n=6)$ and in the presence of hGSTM2-2 (1.0 $\pm 0.2 \mathrm{~s}, n=6)$ (Fig. 7). The decay of the current at $+60 \mathrm{mV}$ became very rapid so that it frequently occurred during the capacitive transient and could not be accurately measured. Thus, data for $+60 \mathrm{mV}$ reflects only a slower population of inactivation times, which were on average $3.8 \pm 1.7 \mathrm{~s}(n=8)$ in the absence of hGSTM2-2 or $1.7 \pm 0.3(n=7)$ in the presence of the protein (Fig. 7). The faster inactivation in the presence of hGSTM2-2 was correlated with the concentration of hGSTM2-2 (Fig. 8).

The increase in activity at negative potentials occurred between 0.5 and $10 \mathrm{~s}$ after the voltage pulse. It is likely that 

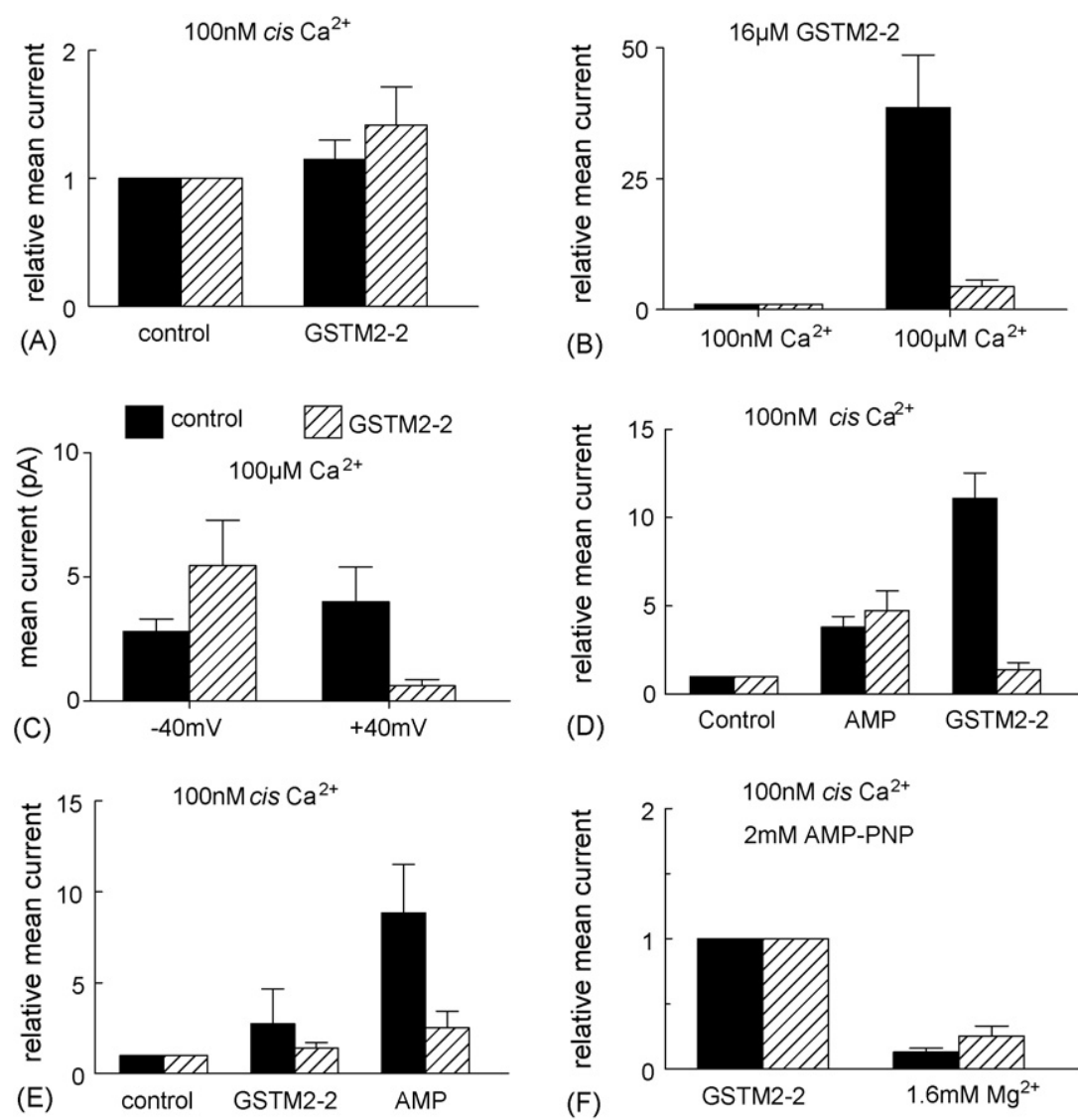

Fig. 4. The effects of hGSTM2-2 are seen only when the RyR1 channels are activated. Average data in each histogram is given for $-40 \mathrm{mV}$ (filled bins) or $+40 \mathrm{mV}$ (hatched bins). (A) hGSTM2-2 $(16 \mu \mathrm{M})$ does not affect channels that are relatively inactive in the presence of a resting cytoplasmic $(\mathrm{cis})\left[\mathrm{Ca}^{2+}\right]$ of $100 \mathrm{nM}$. (B) Channels exposed to $16 \mu \mathrm{M}$ hGSTM2-2 in the presence of $100 \mathrm{nM}$ cis $\mathrm{Ca}^{2+}$ are activated by a subsequent increase in cytoplasmic Ca ${ }^{2+}$ concentration to $100 \mu \mathrm{M}$. (C) Channel activity when cis $\left[\mathrm{Ca}^{2+}\right]$ is increased to $100 \mu \mathrm{M}$ is greater at $-40 \mathrm{mV}$ when hGSTM2-2 is also present, or depressed at $+40 \mathrm{mV}$. (D) AMP-PNP $(2 \mathrm{mM})$ added in the presence of $100 \mathrm{nM}$ cis $\mathrm{Ca}^{2+}$ causes an $\sim 2$-fold increase in relative mean current of RyR1 at both +40 and $-40 \mathrm{mV}$. hGSTM2-2 $(16 \mu \mathrm{M})$ then caused an increase in activity of channels activated by AMP-PNP at $-40 \mathrm{mV}$ and a decrease in activity at $+40 \mathrm{mV}$. (E) As in (A), $16 \mu \mathrm{M}$ hGSTM2-2 did not significantly alter the activity of RyR1 channels with $100 \mathrm{nM}$ cis $\mathrm{Ca}^{2+}$. After subsequent addition of 2 mM AMP-PNP, channel activity increased at both $+40 \mathrm{mV}$ and $-40 \mathrm{mV}$, but the increase was significantly greater at $-40 \mathrm{mV}$ than at $+40 \mathrm{mV}$. The final relative mean current was similar when hGSTM2-2 was added either before (E) or after (D) AMP-PNP. (F) $1.6 \mu \mathrm{M} \mathrm{Mg}^{2+}$ strongly inhibits channels modified by cis hGSTM2-2 in the presence of $100 \mathrm{nM} \mathrm{Ca}^{2+}$ and $2 \mathrm{mM}$ AMP-PNP in the cis solution.

this time-dependence reflected the time taken for the inactivation that was established at the positive potential to decay once the potential was reversed. The increase in activity at the negative potential could be seen only after inactivation was removed. Two important differences between channel activity in the absence and presence of hGSTM2-2 need further mention. Firstly, the delay before the onset of activity with a voltage step from positive to negative potentials was rarely seen under control conditions. It may well be that inactivation at positive potentials was weak under control conditions and that recovery from inactivation occurred very rapidly (within a few ms) after the voltage step and could not be clearly resolved. The second difference was that a voltage step-dependent inactivation was observed at negative potentials in 11 of 12 experiments under control conditions, but was not seen in any experiments at negative potentials after addition of hGSTM2-2, even though activity at this potential was enhanced. hGSTM2-2, while enhancing inactivation at positive potential, prevented inactivation at negative potentials.
In conclusion, the overall effect of hGSTM2-2 was consistent with the GST protein (a) activating RyR1 channels, (b) enhancing the onset of an activation-dependent inactivation process at positive potentials and (c) decreasing the rate of recovery from inactivation when the bilayer potential was reversed.

\section{4. hGSTM2-2 inhibits cardiac RyR2 channels}

We have previously reported that GST and CLIC proteins have a RyR isoform specific action, increasing RyR 1 channel activity, but inhibiting RyR2 channels [4-6]. hGSTM2-2 followed the same pattern. The inhibition of RyR2 occurred at $>10$-fold lower concentrations of hGSTM2-2, than the activation of RyR1 (Fig. 9). Increasing hGSTM2-2 concentrations to 15-30 $\mu \mathrm{M}$ did not cause further inhibition and no reflection of the activation (seen in RyR1 channels) could be observed. The RyR 2 channels were $50 \%$ inhibited by $<1 \mu$ M hGSTM22 in the cis chamber at both positive and negative potentials. 

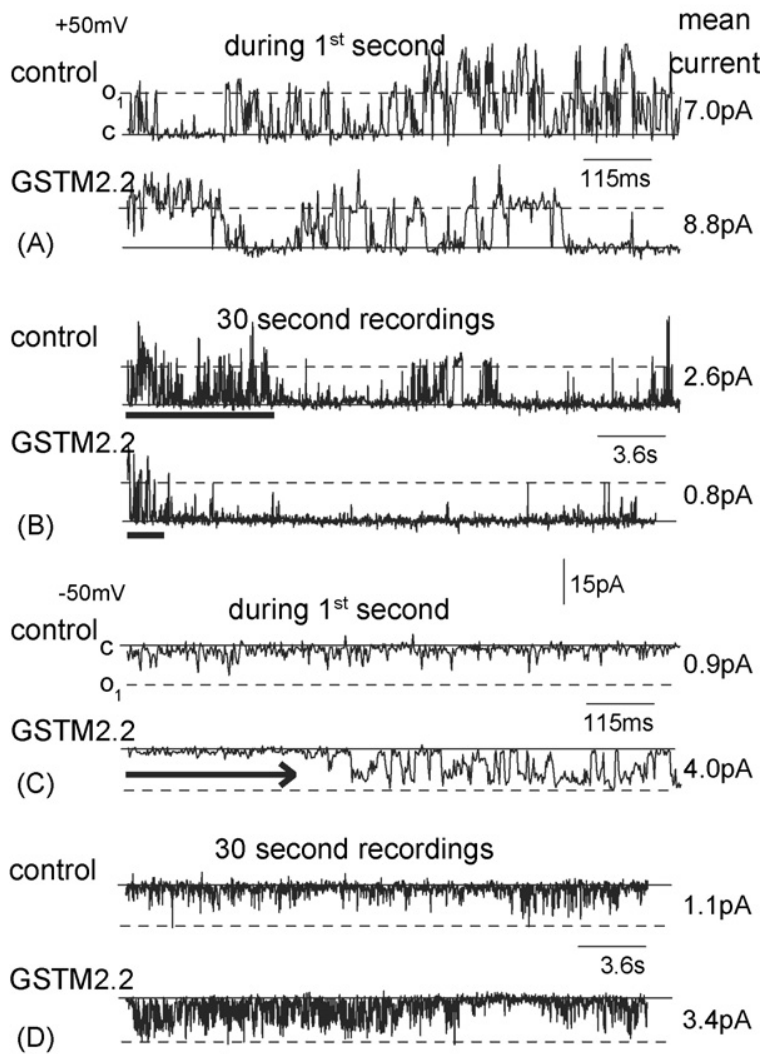

Fig. 5. Effects of hGSTM2-2 on RyR1 activity vary with time after the voltage pulse. Bilayer potential was switched between +50 and $-50 \mathrm{mV}$ every $30 \mathrm{~s}$. Each trace begins within $300 \mathrm{~ms}$ of the voltage step. The mean current during the trace is given to the right of each trace. (A and B) At $+50 \mathrm{mV}$. (A) Activity during the first second after the voltage step is greater in the presence hGSTM2-2 than in its absence. (B) In contrast to the first second, activity over the $30 \mathrm{~s}$ record was less with hGSTM2-2 than in the absence of the protein because activity decayed rapidly after the voltage step. The bar beneath the records indicates inactivation time, which is briefer in the presence of hGSTM2-2. (C and D) At $-50 \mathrm{mV}$. Channel activity during the first second after the voltage change (C) was the same or less with hGSTM22 than control. There is a distinct delay (arrow) before the onset of activity after the voltage step to $-50 \mathrm{mV}$. Activity during the 30 s period at $-50 \mathrm{mV}$ (D) was greater with hGSTM2-2 than control. Channel opening is upward at $+50 \mathrm{mV}$, or downward at $-50 \mathrm{mV}$, from the closed level, c, to the open current for a single channel, $o_{1}$, and higher levels when more than one channel is open.
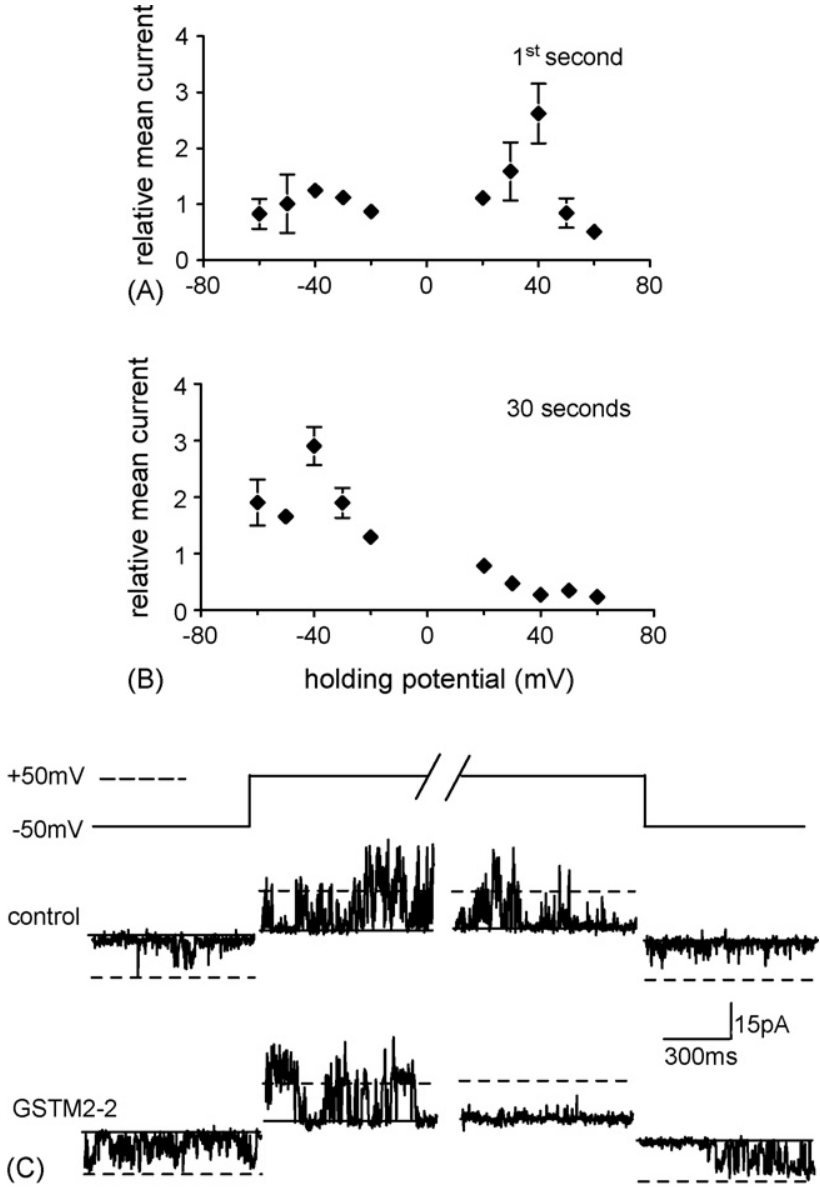

Fig. 6. Voltage- and time-dependent effects of hGSTM2-2 on RyR1 activity. Average relative mean current as a function of potential, during the first second after the voltage step (A) and during the 30 s recording (B). Channel activity at negative potentials is close to control during the first second, but enhanced by hGSTM2-2 during the $30 \mathrm{~s}$ period. Conversely at positive potentials, activity is greater than control with GSTM2-2 during the first second, but depressed over the $30 \mathrm{~s}$ period. (C) Examples of channel activity immediately before and shortly $(\sim 300 \mathrm{~ms}-1 \mathrm{~s})$ after changes in potential between -50 and $+50 \mathrm{mV}$. Under control conditions, activity was generally greater at $+50 \mathrm{mV}$ than at $-50 \mathrm{mV}$ and a little greater initially at $+50 \mathrm{mV}$ than after $30 \mathrm{~s}$. The potential-dependent differences were established within $300 \mathrm{~ms}$ of the voltage step. In the presence of hGSTM2-2, activity at the start of the 30 s recording at $+50 \mathrm{mV}$ was greater than control, but was significantly less than control after $30 \mathrm{~s}$. Activity at $-50 \mathrm{mV}$ with hGSTM2-2 was less than control during the first $\sim 350 \mathrm{~ms}$ and then significantly greater than control for the remainder of the record. Noticeable is the delay after the voltage step from +50 to $-50 \mathrm{mV}$ before activity increased.

Table 2

Relative open probability for RyR1 channels exposed to $30 \mu \mathrm{M}$ of GST isolated from skeletal muscle (skeletal GST), and RyR2 channels exposed to $15 \mu \mathrm{M}$ of GST isolated from cardiac muscle (cardiac GST)

\begin{tabular}{llllll}
\hline & \multicolumn{2}{l}{ Skeletal GST } & & \multicolumn{2}{l}{ Cardiac GST } \\
\cline { 2 - 3 } \cline { 5 - 6 } & $+40 \mathrm{mV}$ & $-40 \mathrm{mV}$ & & $+40 \mathrm{mV}$ & $-40 \mathrm{mV}$ \\
\hline RyR1 & $1.99 \pm 0.53(n=4)$ & $1.98 \pm 0.25^{*}(n=4)$ & $5.33(n=1)$ & $2.6(n=1)$ \\
RyR2 & $0.062(n=1)$ & $0.14(n=2)$ & $0.055 \pm 0.03^{*}(n=4)$ & $0.093 \pm 0.06^{*}(n=4)$ \\
\hline
\end{tabular}

\footnotetext{
* indicates a significant change in open probability from the control value.
} 

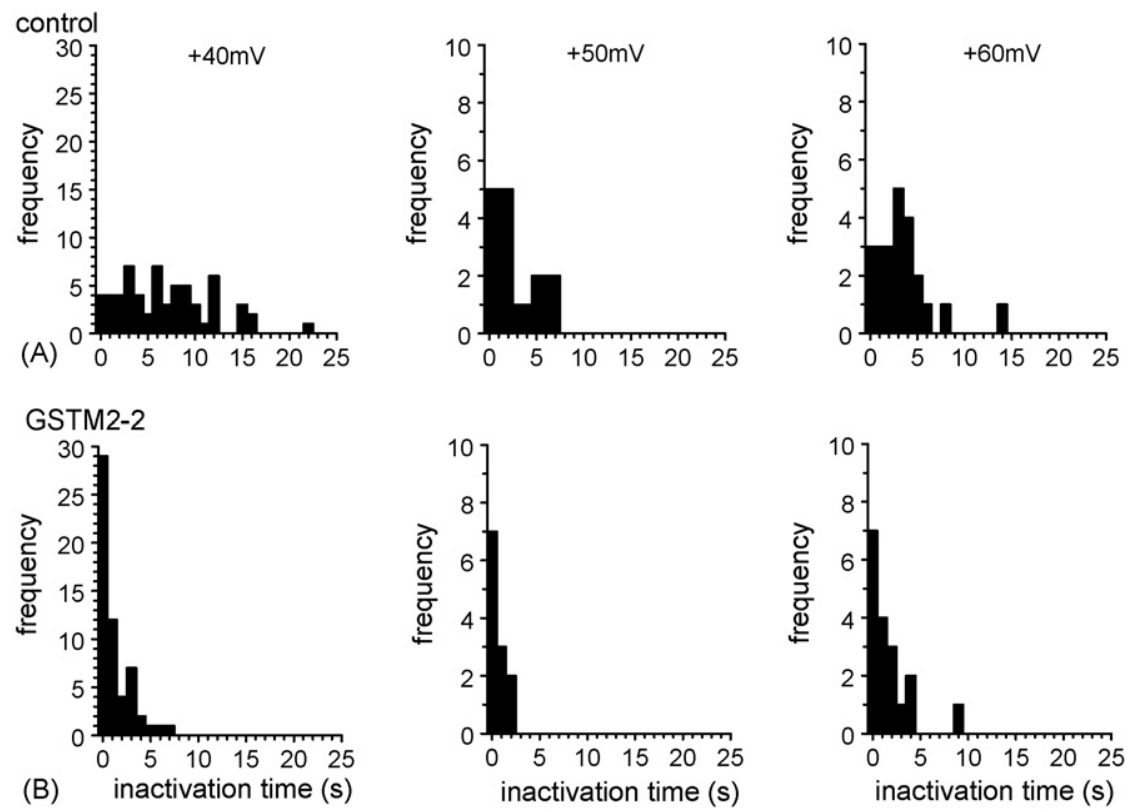

Fig. 7. Inactivation of skeletal RyR1 channels becomes faster in the presence of hGSTM2-2 and inactivation times are briefer when the bilayer potential is more positive under all conditions. (A) Distribution of inactivation times under control conditions (before addition of hGSTM2-2) at $+40,+50$ and $+60 \mathrm{mV}$. (B) Distribution of inactivation times after addition of hGSTM2-2 at $+40,+50$ and $+60 \mathrm{mV}$.

The fall in activity was readily reversible when the GST was perfused out of the cis chamber. The decline in activity was not voltage-dependent and was apparent immediately after the voltage pulse. Thus, the voltage-independent inhibition of RyR2 was very different from the voltage-dependent inactivation of RyR1 at positive potentials.

\subsection{Actions of GST isolated from muscle on RyR channels}

The actions of the total GSTs isolated from skeletal and cardiac muscle (Fig. 1) were examined on RyR1 and RyR2 channels. RyR1 channels were significantly activated by $30 \mu \mathrm{M}$ of the skeletal muscle GST isolate at $-40 \mathrm{mV}$ (Table 2). Thirty micromolar of the cardiac muscle GST isolate activated a low activity skeletal RyR in one experiment. Three of the skeletal RyRs exposed to the skeletal GST isolate were low activity and their relative activity with GST was $2.4 \pm 0.47$. These and the low activity channel exposed to the cardiac isolate had an average $P_{\mathrm{o}}$ of $0.05 \pm 0.024$. The open probability at $+40 \mathrm{mV}$ for one high activity RyR 1 channel fell from 0.18 to 0.13 after exposure to $30 \mu \mathrm{M}$ of the skeletal muscle GST isolate. This channel demonstrated the same voltage-dependent inhibition described for high activity channels exposed to hGSTM2-2 (above).

The cardiac muscle GST isolate reduced the activity of four RyR2 channels at +40 and $-40 \mathrm{mV}$ (Table 2). Since $15 \mu \mathrm{M}$ cardiac GST significantly depressed RyR2 activity, higher concentrations were not used in order to conserve the protein. The relative activity of one channel exposed to $15 \mu \mathrm{M}$ of the skeletal GST isolate fell to 0.14 control. These five RyR2 channels were all high activity with an initial $P_{\mathrm{o}}$ of $0.37 \pm 0.11$. The concentrations of GST isolate required to inhibit RyR2 channels was approximately double that of hGSTM2-2 required for inhibition (above), since the activity of one RyR2 channel was not altered by exposure to 1 or $5 \mu \mathrm{M}$ of the cardiac isolate but fell when the concentration was increased to $15 \mu \mathrm{M}$. It is possible that the Pi class GST present in the cardiac isolate was a lower affinity inhibitor of RyR2 than the GSTM-2 like proteins.

\section{Discussion}

Several novel findings are reported here. Firstly, GSTs are strongly expressed in the cytoplasmic and membrane fractions from rabbit skeletal muscle and sheep heart and a significant fraction of the cytoplasmic GSTs belong to the GST Mu class, probably closely related to human hGSTM2-

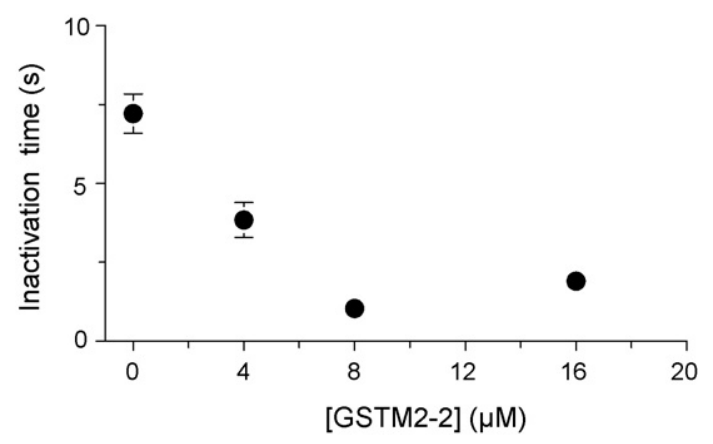

Fig. 8. Inactivation times at $+40 \mathrm{mV}$ are correlated with the concentration of hGSTM2-2 used. Inactivation time is shown as a function of hGSTM2-2 concentration and becomes shorter as hGSTM2-2 concentration increases. 


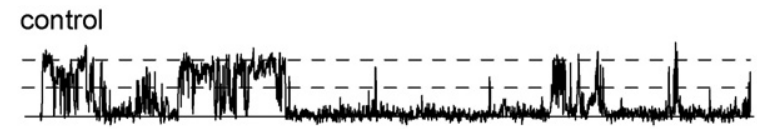

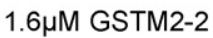
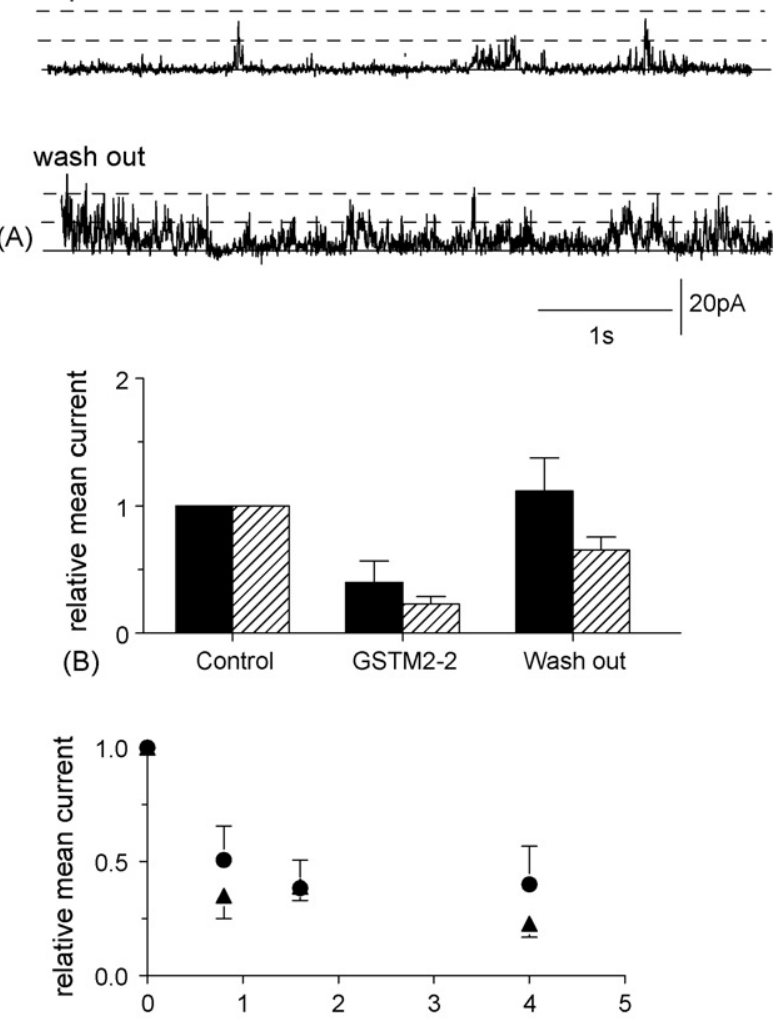

(C)

[GSTM2-2] $(\mu \mathrm{M})$

Fig. 9. hGSTM2-2 depresses the activity of cardiac RyR2 channels at both +40 and $-40 \mathrm{mV}$. (A) Records of channel activity at $+40 \mathrm{mV}$ with $100 \mu \mathrm{M}$ cis $\mathrm{Ca}^{2+}$, under control conditions (upper panel), with 1.6 $\mu \mathrm{M}$ hGSTM2-2 (middle panel) and after washout of the cis solution (lower panel). (B) Average effect of addition and washout of $1.6 \mu \mathrm{M}$ hGSTM2-2 on relative mean current at $-40 \mathrm{mV}$ (filled bins) and $+40 \mathrm{mV}$ (hatched bins). (C) Relative mean current plotted as a function of hGSTM2-2 concentration at $+40 \mathrm{mV}$ (filled circles) and $-40 \mathrm{mV}$ (filled triangles).

2. The cytoplasmic concentration of GSTs in skeletal muscle and heart was of the order of 17-93 $\mu \mathrm{M}$. We have conducted the first in depth study of the actions of a GST on the skeletal muscle RyR and report a novel action of hGSTM2-2 and GSTs isolated from muscle in activating RyR1 channels and in enhancing voltage-dependent inactivation of higher activity RyR1 channels at positive potentials. In contrast, hGSTM2-2 and the GSTs isolated from muscle inhibit cardiac RyR channels in a manner that is independent of voltage, and consistent with reported effects of other members of the GST structural family on RyR2 [4-6].

\subsection{GSTM2 in non-human skeletal and cardiac muscle}

Previous studies of the Mu class of GSTs have shown that the human GSTM2-2 is specifically expressed in human skeletal muscle [11-13]. We now show that a GSTM2-like protein is also abundantly expressed in the cytoplasm of nonhuman rabbit skeletal and sheep cardiac muscle. Because of its abundance in muscle, this GST is likely to have muscle specific actions, which might include the modulation of RyR channels and regulation of $\mathrm{Ca}^{2+}$ signalling by $\mathrm{Ca}^{2+}$ released from the SR. The different actions on skeletal and cardiac RyRs suggest a muscle-type specific role. In skeletal muscle, activation of RyR1 by hGSTM2-2 would enhance $\mathrm{Ca}^{2+}$ release from the SR especially during the initial phase of EC coupling when channels are most active. The time-dependent inhibition dominates at positive potentials, which would tend to develop during $\mathrm{Ca}^{2+}$ release. This inhibition is relieved over several seconds when the potential is reversed. Thus, hGSTM2-2 would act to initially enhance $\mathrm{Ca}^{2+}$ release, but then to turn off release and conserve the SR $\mathrm{Ca}^{2+}$ content during continued activation.

There are in fact several mechanisms, which ensure that skeletal RyRs are minimally active when muscle is not contracting. These include channel inhibition by $\mathrm{Mg}^{2+}$, which is relieved by voltage-sensor activation during EC coupling [30-32]. Cardiac RyRs on the other hand have a lower affinity for $\mathrm{Mg}^{2+}$-inhibition [32] and thus require other endogenous inhibitors to prevent excess $\mathrm{Ca}^{2+}$ release from the SR. Therefore in cardiac muscle, the inhibitory action of hGSTM2-2 on RyR2 would act to conserve $\mathrm{Ca}^{2+}$ under all conditions.

The fact that there are several classes of GST proteins expressed in both skeletal and cardiac muscle, all of which appear to influence RyR1 and RyR2 channels, means that the action of the proteins on muscle contraction is protected from polymorphisms which might reduce the activity of any one individual member of the class. Thus the similar actions of the different GSTs on the $\mathrm{Ca}^{2+}$ release channel, particularly the cardiac RyR, means that function is conserved even if one member of the class is disabled. For example, in humans the GSTM1 gene is deleted in about $50-60 \%$ of Europeans [33]. Indeed it has been shown in mice that knockout of one member of the GST family (GSTZ1-1) is accompanied by enhanced expression of other members of the GST family, including a member of the Mu class [34]. The concentration of GSTM2-like protein in the cytoplasm is of the order of $17-93 \mu \mathrm{M}$, which is in the range of concentrations that maximally alter RyR activity in both muscle types.

The fact that the activity of both cardiac and skeletal RyR channels can be modulated by GSTs raises the possibility that some effects of recombinant GST-fusion proteins on RyR channels could be attributed to a direct effect of the GST on the channel. This should perhaps be taken into consideration when evaluating the effects of the fusion protein by also examining the action of the GST alone. The GST normally used in expression vectors is $\mathrm{Sj} 26$ from Schistosoma japonicum. We have not systematically examined the actions of this GST on RyR1 or RyR2.

The recombinant hGSTM2-2 used in the present experiments is purified by glutathione agarose affinity chromatog- 
raphy and is eluted from the affinity matrix with $10 \mathrm{mM}$ reduced glutathione. The active site is likely to be saturated with glutathione at this point and because of its high affinity for glutathione it is unlikely to be washed out during dilution in the bilayer experiments. In our experience it is extremely difficult to obtain GSTs that are free of GSH, so we assume that GSH was bound to both the hGSTM2-2 and to the GSTs isolated from skeletal and cardiac muscle.

\subsection{Voltage-dependent inactivation in RyRI}

Voltage-dependent inactivation is normally observed in a subpopulation of both RyR1 and RyR2 channels at both positive and negative potentials [17,25-29]. A similar inactivation is apparent at a constant voltage if channels are activated by a rapid step in $\mathrm{Ca}^{2+}$ concentration. Thus, the term "voltage-dependent inactivation" is somewhat misleading because the inactivation process follows rapid activation and is independent of the means of activation. The function of this inactivation is thought to provide a safety valve, which prevents maintained activation over long periods of time [27]. Indeed, inactivation in cardiac RyRs provides a mechanism for termination of $\mathrm{Ca}^{2+}$ release [29]. Curiously, the voltage-dependence of inactivation can be modified by drugs. Application of DIDS increases the activity of RyR2 channels at both positive and negative potentials, but inactivation is apparent only at negative potentials [29]. These authors suggest that "the particular ligand activating the channel can change the voltage-dependence of the channel in a characteristic manner and can set the voltage at which the channel will inactivate". In this study, we provide another example of a potential-dependent modification of inactivation. The modification by hGSTM2-2 differs from the DIDS-modification in that it is specific for RyR 1 and ensures that inactivation occurs primarily at positive potentials. The inactivation also differs in that recovery after the potential is reversed is noticeably slower in the presence of the GST.

The dependence of inactivation on channel open probability rather than bilayer potential per se [27], suggests that inactivation cannot depend on charge reorientation alone, but must also be linked to a non voltage-dependent activation gate. Perhaps charged residues associated with the inactivation process can move after a voltage step and also move in response to non-voltage dependent conformational changes that occur with strong activation by $\mathrm{Ca}^{2+}$, DIDS or hGSTM22. The data are consistent with a hypothesis in which (a) $\mathrm{Ca}^{2+}$ or AMP-PNP activate the RyR and do not restrict the movement of the charged residues that leads to inactivation, (b) DIDS binding restricts the charge movement and inactivation at positive potentials, while (c) hGSTM2-2 binding enhances inactivation at positive potentials and restricts inactivation of charge movement so as to prevent inactivation at negative potentials. The interaction sites for hGSTM2-2 may well be different in RyR1 and RyR2, since hGSTM2-2 causes a non-voltage-dependent inhibition of RyR2. Alternatively, the gating mechanism linked to the binding site could differ in the two RyR isoforms. These possibilities require further investigation.

In conclusion, the results show novel actions of the muscle-type hGSTM2-2 on skeletal muscle RyR1, which unmask properties of the voltage-dependent inactivation mechanism and reveal a novel slow recovery from inactivation. The results unmask a ligand binding specific modification of the voltage-dependent inactivation process that is available to skeletal but not cardiac RyRs. Although the action of hGSTM2-2 on cardiac and skeletal RyR appears to differ, the overall inhibitory action under conditions likely to exist during $\mathrm{Ca}^{2+}$ release, suggest that the GST acts to conserve $\mathrm{Ca}^{2+}$ in the sarcoplasmic reticulum of both skeletal and cardiac muscle.

\section{Acknowledgements}

We thank Suzy Pace and Joan Stivala for assistance with SR vesicle preparation. The work was supported by grant \#268027 from the National Health and Medical Research Council.

\section{References}

[1] G. Meissner, Ryanodine receptor/ $\mathrm{Ca}^{2+}$ release channels and their regulation by endogenous effectors, Ann. Rev. Physiol. 56 (1994) 485-508.

[2] G. Meissner, Regulation of mammalian ryanodine receptors, Front. Biosci. 7 (2002) d2072-d2080.

[3] N.A. Beard, D.R. Laver, A.F. Dulhunty, Calsequestrin and the calcium release channel of skeletal and cardiac muscle, Prog. Biophys. Mol. Biol. 85 (2004) 33-69.

[4] P.G. Board, M. Coggan, S. Watson, P.W. Gage, A.F. Dulhunty, CLIC2 modulates cardiac ryanodine receptor $\mathrm{Ca}^{2+}$ release channels, Int. J. Biochem. Cell Biol. 36 (2004) 1599-1612.

[5] A. Dulhunty, P. Gage, S. Curtis, G. Chelvanayagam, P. Board, The glutathione transferase structural family includes a nuclear chloride channel and a ryanodine receptor calcium release channel modulator, J. Biol. Chem. 276 (2001) 3319-3323.

[6] A.F. Dulhunty, P. Pouliquin, M. Coggan, P.W. Gage, P.G. Board, A recently identified member of the glutathione transferase structural family modifies cardiac RyR2 substate activity, coupled gating and activation by $\mathrm{Ca}^{2+}$ and ATP, Biochem. J. 390 (2005) 333343.

[7] P.G. Board, M. Coggan, G. Chelvanayagam, et al., Identification, characterization, and crystal structure of the Omega class glutathione transferases, J. Biol. Chem. 275 (2000) 24798-24806.

[8] S.G. Cho, Y.H. Lee, H.S. Park, et al., Glutathione $S$-transferase mu modulates the stress-activated signals by suppressing apoptosis signalregulating kinase 1, J. Biol. Chem. 276 (2001) 12749-12755.

[9] J.M. Fernandez-Canon, M.A. Penalva, Characterization of a fungal maleylacetoacetate isomerase gene and identification of its human homologue, J. Biol. Chem. 273 (1998) 329-337.

[10] V. Adler, Z. Yin, S.Y. Fuchs, et al., Regulation of JNK signalling by GSTp, EMBO J. 18 (1999) 1321-1334.

[11] T. Suzuki, M. Coggan, D.C. Shaw, P.G. Board, Electrophoretic, Electrophoretic and immunological analysis of human glutathione $S$-transferase isozymes, Ann. Hum. Genet. 51 (Part 2) (1987) 95106.

[12] P.G. Board, T. Suzuki, D.C. Shaw, Human muscle glutathione $S$ transferase (GST-4) shows close homology to human liver GST-1, Biochim. Biophys. Acta 953 (1988) 214-217. 
[13] A.J. Hussey, L.A. Kerr, A.D. Cronshaw, D.J. Harrison, J.D. Hayes, Variation in the expression of Mu-class glutathione $S$-transferase isoenzymes from human skeletal muscle. Evidence for the existence of heterodimers, Biochem. J. 273 (Part 2) (1991) 323-332.

[14] A. Saito, S. Seiler, A. Chu, S. Fleischer, Preparation and morphology of sarcoplasmic reticulum terminal cisternae from rabbit skeletal muscle, J. Cell Biol. 99 (1984) 875-885.

[15] G.P. Ahern, P.R. Junankar, A.F. Dulhunty, Single channel activity of the ryanodine receptor calcium release channel is modulated by FK-506, FEBS Lett. 352 (1994) 369-374.

[16] B.K. Chamberlain, S. Fleischer, Isolation of canine cardiac sarcoplasmic reticulum, Methods Enzymol. 157 (1988) 91-99.

[17] D.R. Laver, L.D. Roden, G.P. Ahern, K.R. Eager, P.R. Junankar, A.F. Dulhunty, Cytoplasmic $\mathrm{Ca}^{2+}$ inhibits the ryanodine receptor from cardiac muscle, J. Membr. Biol. 147 (1995) 7-22.

[18] V.L. Ross, P.G. Board, Molecular cloning and heterologous expression of an alternatively spliced human Mu class glutathione $S$-transferase transcript, Biochem. J. 294 (Part 2) (1993) 373-380.

[19] H. Towbin, T. Staehelin, J. Gordon, Electrophoretic transfer of proteins from polyacrylamide gels to nitrocellulose sheets: procedure and some applications, Proc. Natl. Acad. Sci. U.S.A. 76 (1979) 43504354.

[20] E.W. Minium, B.M. King, G. Bear, Statistical Reasoning in Psychology and Education, John Wiley and Sons, New York, 1993.

[21] A.J. Hussey, J.D. Hayes, Human Mu-class glutathione $S$-transferases present in liver, skeletal muscle and testicular tissue, Biochim. Biophys. Acta 1203 (1993) 131-141.

[22] N.A. Curtin, J.C. Clapham, C.J. Barclay, Excess recovery heat production by isolated muscles from mice overexpressing uncoupling protein-3, J. Physiol. 542 (2002) 231-235.

[23] B.R. Eisenberg, Quantitative Ultrastructure of Mammalian Skeletal Muscle, American Physiological Society, Bethesda, 1983.
[24] J.J. Marengo, C. Hidalgo, R. Bull, Sulfhydryl oxidation modifies the calcium dependence of ryanodine-sensitive calcium channels of excitable cells, Biophys. J. 74 (1998) 1263-1277.

[25] A.L. Percival, A.J. Williams, J.L. Kenyon, M.M. Grinsell, J.A. Airey, J.L. Sutko, Chicken skeletal muscle ryanodine receptor isoforms: ion channel properties, Biophys. J. 67 (1994) 1834-1850.

[26] J. Ma, Desensitization of the skeletal muscle ryanodine receptor: evidence for heterogeneity of calcium release channels, Biophys. J. 68 (1995) 893-899.

[27] D.R. Laver, G.D. Lamb, Inactivation of $\mathrm{Ca}^{2+}$ release channels (ryanodine receptors RyR1 and RyR2) with rapid steps in $\left[\mathrm{Ca}^{2+}\right]$ and voltage, Biophys. J. 74 (1998) 2352-2364.

[28] R. Sitsapesan, A.J. Williams, Do inactivation mechanisms rather than adaptation hold the key to understanding ryanodine receptor channel gating? J. Gen. Physiol. 116 (2000) 867-872.

[29] A.P. Hill, R. Sitsapesan, DIDS modifies the conductance, gating, and inactivation mechanisms of the cardiac ryanodine receptor, Biophys. J. 82 (2002) 3037-3047.

[30] G.D. Lamb, D.G. Stephenson, Effect of $\mathrm{Mg}^{2+}$ on the control of $\mathrm{Ca}^{2+}$ release in skeletal muscle fibres of the toad, J. Physiol. 434 (1991) 507-528.

[31] G.D. Lamb, D.G. Stephenson, Effects of intracellular pH and $\left[\mathrm{Mg}^{2+}\right]$ on excitation-contraction coupling in skeletal muscle fibres of the rat, J. Physiol. 478 (Part 2) (1994) 331-339.

[32] D.R. Laver, T.M. Baynes, A.F. Dulhunty, Magnesium inhibition of ryanodine-receptor calcium channels: evidence for two independent mechanisms, J. Membr. Biol. 156 (1997) 213-229.

[33] P.G. Board, Biochemical genetics of glutathione-S-transferase in man, Am. J. Hum. Genet. 33 (1981) 36-43.

[34] A.C. Blackburn, K.I. Matthaei, C. Lim, et al., Deficiency of glutathione transferase zeta causes oxidative stress and activation of antioxidant response pathways, Mol. Pharmacol. 69 (2006) 650-657. 